\title{
Um Sistema para Classificação de Objetos de Aprendizagem MCS - Multimodality Classification System
}

\author{
Alberto Bastos do Canto Filho - alberto.canto@ufrgs.br - PPGIE - UFRGS \\ Thaísa Jacintho Müller - thaisa.muller@pucrs.br - PPGIE - UFRGS - PUCRS \\ Érico M. H. Amaral - erico.amaral@unipampa.edu.br - PPGIE - UFRGS -UNIPAMPA \\ José Valdeni de Lima - valdeni@inf.ufrgs.br - PPGIE - UFRGS \\ Liane Margarida Rockenbach Tarouco - liane@penta.ufrgs.br - PPGIE - UFRGS
}

Resumo: Este artigo apresenta o extrato de uma pesquisa que resultou no aplicativo denominado MCS - Multimodality Classification System, implementado com o intuito de auxiliar professores na busca e seleção de materiais a serem utilizados com seus alunos, considerando seus objetivos educacionais. Tal sistema tem a finalidade de classificar os níveis de multimodalidade presentes em partes ou como um todo em objetos de aprendizagem. O método aplicado pelo software leva o usuário a identificar o OA por meio de categorias preestabelecidas, algumas das quais ineficazes para o aprendizado e outras que apontam para um apoio efetivo na construção do conhecimento. Nesta etapa da pesquisa, o MCS foi testado em cinco objetos de aprendizagem, mostrando sua eficácia e possível aplicabilidade com professores (ou mesmo alunos) em geral.

Palavras Chave - multimodalidade, objetos de aprendizagem, classificação

Abstract: This article presents the extract of a research which resulted on the application called MCS - Multimodality Classification System, implemented with the aim of helping teachers on seeking and choosing material to be used with their students, regarding their educational goals. This system has the aim of rating the levels of multimodality included in some parts of them or as a whole in learning objects. The method applied by the software leads the user to identify the OA through preestablished categories, some ineffective for learning and some that link to an effective support on building knowledge. In this step, MCS has been tested in five learning objects, showing its effectiveness and possible applicability with teachers (or even students) in general.

Keywords - multimodality, learning objects, classification

\section{Introdução}

Objetos de aprendizagem (OAs) são recursos que vêm sendo amplamente utilizados no ensino, dada sua versatilidade, dinamicidade, reusabilidade, entre outras vantagens que trazem em relação aos materiais instrucionais ditos tradicionais.

Mais do que estudos específicos sobre concepção e desenvolvimento de OAs, a sua disseminação trouxe também uma preocupação sobre avaliação dos objetos disponíveis na rede ou em outros meios. Inicia-se, assim, um processo de estabelecimento de critérios de qualidade que auxiliem professores e alunos na seleção de materiais alinhados aos seus objetivos educacionais. 
Tanto os projetos como a seleção de materiais existentes devem passar por algum tipo de avaliação de eficácia, que permita identificar a potencial contribuição para que os objetivos educacionais (Bloom, 1956) sejam alcançados. A medida direta da eficácia é um processo complexo e com custos elevados, que somente é viável em ambientes de pesquisa. Tal medição se torna inviável devido aos seguintes motivos: (1) é necessário envolver estudantes que representem adequadamente o público alvo e aplicar testes que avaliem a aprendizagem. Este procedimento necessita um grande número de horas e um grande número de pessoas, portanto tem um alto custo; (2) não é possível ser realizada antes da conclusão do projeto do OA; (3) caso o procedimento sinalize que o OA não é eficaz, todos os recursos investidos no desenvolvimento e avaliação terão sido desperdiçados.

Avaliações subjetivas podem e devem ser utilizadas, considerando o potencial do OA como material potencialmente significativo, capaz de contribuir para que o estudante estabeleça relações substantivas não arbitrárias entre as novas proposições e os seus conceitos subsunçores (aprendizado significativo - Ausubel, Novak e Hanesian, 1978). Adicionalmente à avaliação subjetiva, alguns aspectos técnicos podem ser avaliados de forma simples e objetiva, verificando se o OA foi especificado de acordo com determinados princípios. Já no caso da verificação multimodalidade, por exemplo, tem-se uma avaliação mais complexa, devido à existência de diversas formas de multimodalidade possíveis.

Neste contexto, o presente trabalho apresenta uma proposta para sistematizar a classificação de OAs de acordo com o tipo de multimodalidade. Sendo assim, na seção

2 é apresentado um referencial teórico, que trata de aprendizagem e multimodalidade, trazendo também a proposta inicial de classificação e trabalhos correlatos; a seção 3 versa sobre a metodologia utilizada, mostrando a criação de um fluxograma que evoluiu ao aplicativo citado; a seção 4 trata da implementação do aplicativo e, nas seções 5 e 6, são apresentados resultados e conclusões obtidas com a pesquisa até o presente momento.

\section{Referencial Teórico}

Para embasar a proposta de classificação de OAs quanto ao nível de multimodalidade, é apresentado aqui um conjunto de referências que serviram de base para este estudo.

\subsection{Aprendizagem e Multimodalidade}

Como primeiro tópico da teoria a ser estudada, elencou-se o conceito de multimodalidade, o qual pode variar de acordo com o autor utilizado. Após uma revisão destas possibilidades, a multimodalidade será definida aqui como a característica dos ambientes de aprendizagem que apresentam o conteúdo abordado utilizando simultaneamente dois modos de apresentação: verbal e não verbal (Paivio, 1986). Destaca-se ainda que a multimodalidade é um recurso com ganhos potenciais de eficácia, principalmente na aprendizagem receptiva, defendida por Ausubel, Novak e Hanesian (1978) como a forma mais simples e eficiente de aquisição de conhecimento.

Por outro lado, a eficácia dos sistemas multimodais tem sido fundamentada em estudos sobre os estilos individuais dos estudantes e na teoria da carga cognitiva. Os autores Felder e Brent (2005) definem estilo de aprendizagem como a forma característica com que os estudantes tomam e processam a informação. Grimley e Riding (2009) e Riding e Cheema (1991) utilizam a expressão estilo cognitivo para 
designar a abordagem que o indivíduo normalmente utiliza quando processa uma informação. Uma abordagem comum entre pesquisadores nesta área é procurar isolar fatores independentes (dimensões) que caracterizem os estilos de aprendizagem. Por exemplo, Riding e Cheema (1991) propõem um sistema que considera duas dimensões: (1) Dimensão verbal-imaginária: identifica a forma preferencial como o indivíduo representa as informações quando pensa. $\mathrm{O}$ estudante pode preferir pensar utilizando palavras (verbal) ou imagens e abstrações (não verbal), e; (2) Dimensão globalanalítica: identifica a preferência individual por integrar informações como um todo (abordagem integradora) ou separar o todo em suas partes constituintes (abordagem analítica).

Assim, no que se refere a estilos de aprendizagem, a multimodalidade é uma forma de ganhar eficácia atendendo simultaneamente todos os perfis individuais dentro da dimensão verbal/não verbal.

A Teoria da Carga Cognitiva (Sweller, 1988), por sua vez, considera a capacidade de processamento necessária para a aquisição de esquemas e as questões relacionadas aos problemas decorrentes da sobrecarga cognitiva, que ocorre quando o sistema instrucional exige do estudante uma capacidade de processamento acima dos seus limites disponíveis.

Diversos autores (Sweller, 1988; Sweller et. al., 1998; Paas, Renkl e Sweller, 2003; Low e Sweller, 2005) apresentam princípios de projetos fundamentados na Teoria da Carga Cognitiva, que é descrita por van Merriënboer e Sweller (2010) a partir da arquitetura cognitiva humana (Figura 1). São considerados três tipos de memória: memória sensorial, memória de trabalho e memória de longo prazo.

Já para Baddeley (2010) existem

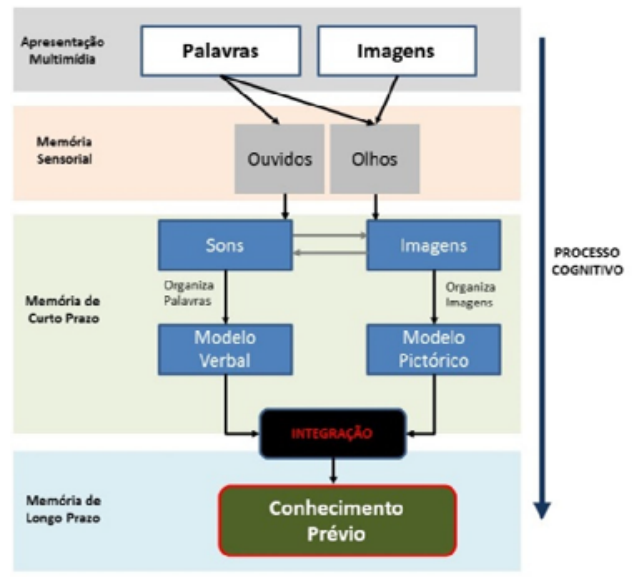

Figura 01. Processamento da Informação dois canais independentes de processamento de informações, associados aos modos sensoriais individuais: um canal para lidar com esquemas visuais ou espaciais e outro canal para lidar com as informações auditivas, especialmente a voz. O uso simultâneo destes dois canais possibilita o tratamento de um número maior de elementos de informação, o que está em consonância com as propostas de multimodalidade (Sung e Mayer, 2012) e com o princípio de projeto multimídia (Mayer, 2005).

Assim, com relação à teoria da carga cognitiva, a multimodalidade é uma forma concreta de minimizar o risco de sobrecarga cognitiva.

\subsection{Objetos Multimodais e sua classificação}

Com a intenção de criar um padrão de classificação de OAs considerando-se aspectos de multimodalidade, criou-se uma tabela de possibilidades que contemplam qualquer objeto, observando-se a existência (ou não) de áudios verbais e não verbais ou de elementos visuais verbais e não verbais e a coerência entre eles. Devido ao grande número de possibilidades, realizou-se um agrupamento das situações similares, dando origem às seguintes classes, apresentadas por Canto et. al. (2013): Classe 1: 
Multimodalidade Dispersiva, ocorre quando uma ou mais mídias apresentam conteúdo que não contribuam para o objetivo educacional proposto ou para a motivação do estudante, gerando carga cognitiva que não contribua para o aprendizado; Classe 2: Não Multimídia, refere-se àqueles objetos que apresentam apenas áudio ou apenas imagem, porém em ambos os modos, para que possa ser considerado multimodal; Classe 3: Texto Sonorizado, compreende os OAs que trazem apenas textos escritos acompanhados de um áudio não verbal; Classe 4: Texto Sonorizado Ilustrado, corresponde aos objetos que contêm textos escritos acompanhados de áudio. Além disto, esta classe apresenta estímulos visuais não verbais, ou seja, o texto sonorizado é acompanhado de uma figura, por exemplo; Classe 5: Redundância Verbal, assim denominada por conter a mesma informação verbal tanto no canal de áudio como na forma visual (texto narrado e escrito), tratando-se de uma alternativa menos eficaz do que a utilização de um único canal (ou narrado ou escrito) (Mayer, Heiser e Lonn, 2001) ; Classe 6: Multimídia Monotônico, compreende basicamente aqueles objetos de aprendizagem que trazem no canal de áudio a informação verbal (palavras faladas) e na tela (visual) as informações não verbais, o que costuma ser bastante satisfatório no cumprimento de seus objetivos educacionais, e; Classe 7: Multimídia Efetivo, considerada a mais adequada segundo a classificação proposta, a qual difere da classe anterior pela preocupação em acrescentar elementos afetivos coerentes com os objetivos educacionais e capazes de motivar o estudante para o aprendizado.

Destaca-se ainda que, caso o objeto não seja multimodal, será classificado como Classe 0 , uma vez que todas as demais são baseadas na multimodalidade. É importante ressaltar que o usuário deverá ser muito cuidadoso para classificar um OA em uma destas classes, uma vez que dependerá de sua percepção a decisão sobre existência ou não dos elementos considerados. Desta forma, ainda que se tenha criado um aplicativo a fim de facilitar este processo, alguns problemas podem persistir.

Na primeira fase da pesquisa, relatada por Canto et. al. (2013), constatou-se que nem sempre é possível classificar um objeto de aprendizagem como um todo, uma vez que suas partes podem apresentar características diferentes. Nestes casos, sugere-se que sejam analisados separadamente os trechos que possuam características comuns.

\subsection{Estudos Correlatos}

Esta seção descreve duas pesquisas que, juntamente com os estudos já apresentados, podem ser consideradas como alinhados ao problema descrito neste artigo.

O trabalho apresentado por Macêdo, Macêdo e Filho (2007) no Workshop de Informática na Educação, com título "Avaliação de um Objeto de Aprendizagem com Base nas Teorias Cognitivas” aborda as concepções de aprendizagem que podem ser utilizadas por professores para a avaliação de OAs, descrevendo as vantagens destas aplicadas à compreensão de conceitos matemáticos. A experimentação da pesquisa foi realizada com a avaliação de três Objetos de Aprendizagem para saber em qual concepção de aprendizagem os mesmos estão fundamentados.

$\mathrm{Na}$ falta de modelos efetivos para classificação de objetos de aprendizagem, Battistella et. al. (2009) publicaram no Simpósio Brasileiro de Informática na Educação (SBIE) uma pesquisa sobre a dificuldade de classificar as ferramentas de autoria existentes e desta forma apontar quais softwares atendem satisfatoriamente às expectativas dos usuários. Com esta finalidade, algumas ferramentas de autoria foram comparadas e avaliadas segundo critérios específicos. O resultado deste estudo 
possibilitou descrever uma proposta de classificação de OAs tendo como base a tecnologia empregada para sua construção.

\section{Metodologia}

A base metodológica utilizada para o desenvolvimento desta pesquisa pode ser descrita por meio de um conjunto de etapas conforme apresentado a seguir: a primeira fase destinou-se à revisão bibliográfica dos temas norteadores do estudo; em um segundo momento foi realizado o estudo do método de classificação de OAs, com base nos níveis de multimodalidade, sendo feito simultaneamente um levantamento de requisitos de software para tal modelo; a terceira etapa caracterizou-se pela definição de uma estrutura para classificação de objetos de aprendizagem, originando um fluxograma com a descrição de tais passos. Ainda nesta fase foi elencada uma linguagem de programação para o desenvolvimento de um software com o intuito de automatizar o processo de classificação. Por fim, a quarta e última fase vislumbrou a avaliação do programa construído por meio da classificação de diferentes OAs e a apresentação destes como resultados e discussões. A Figura 2 demonstra cada uma das fases descritas.

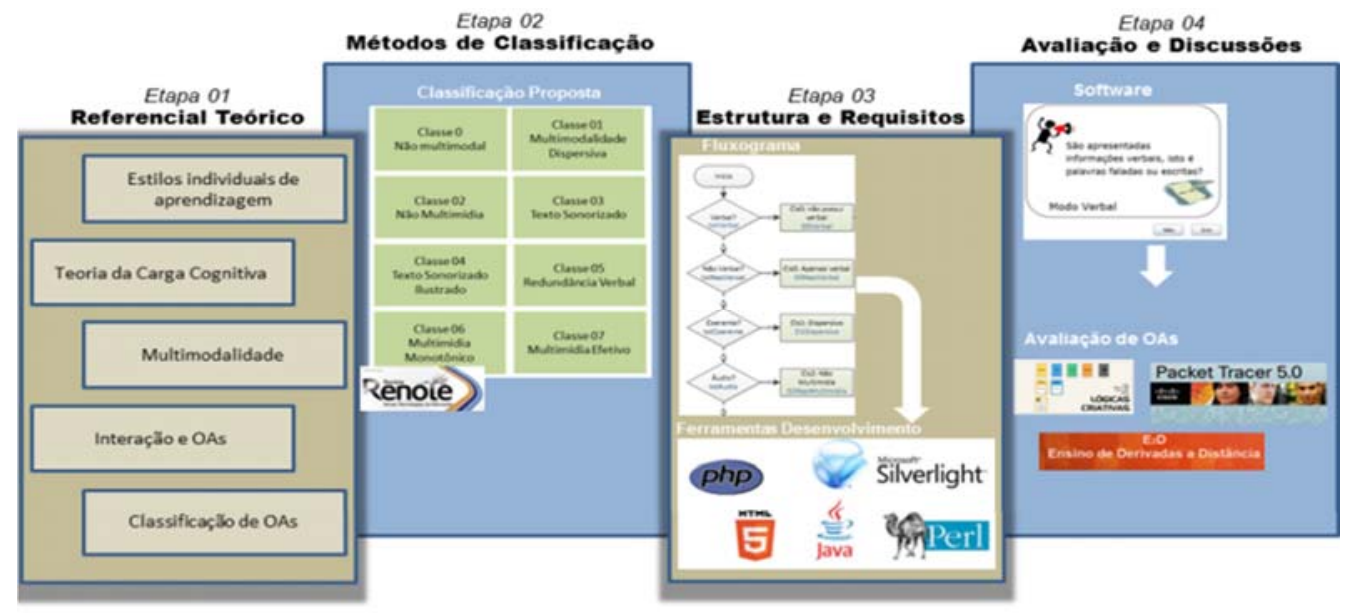

Figura 2. Etapas para o desenvolvimento da Pesquisa

A fim de aumentar a similaridade no agrupamento de um OA, a avaliação de alguns dos resultados obtidos neste estudo permitiram organizar requisitos e implementar um fluxograma que explicita de maneira minuciosa cada elemento de um objeto de aprendizagem e, desta forma, possibilita a descrição do nível de multimodalidade presente no mesmo. A Figura 3 descreve os passos para a observação de um objeto, nos quais os usuários são questionados sobre características como a presença de mídia verbal, não verbal, se os assuntos estão coerentes, se existe áudio e, por fim, se possui características de afetividade. Assim, a classificação pode ser observada do nível zero (não multimodal/Cls0) até o nível sete (Multimodalidade Efetiva/Cls7). 

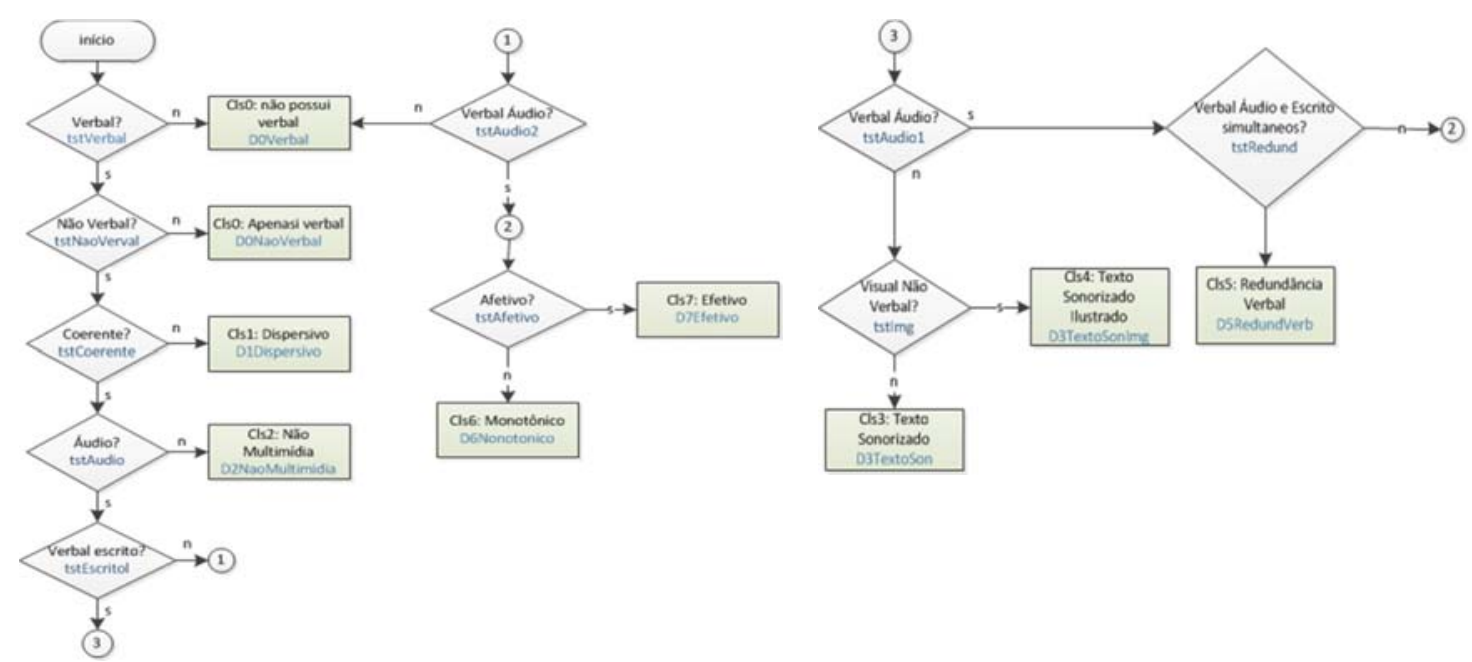

Figura 3. Fluxograma para classificação de OAs

Após a confecção do fluxograma, realizou-se um estudo de softwares de autoria e linguagens de programação passíveis de serem usadas como soluções nesta pesquisa. Ao término da avaliação, o Microsoft Silverlight foi utilizado por se mostrar uma plataforma de desenvolvimento simples, compatível com múltiplos navegadores e com um bom índice de interatividade. Após esta etapa, com a aplicação MCS - Multimodality Classification System em produção, pode-se partir para a etapa de implementação, testes, avaliação e discussão dos resultados.

\section{Implementação}

A metodologia proposta foi utilizada para o desenvolvimento de um aplicativo que tem por objetivo facilitar a análise de objetos de aprendizagem quanto ao seu grau de multimodalidade. Este aplicativo pode ser definido como um metaobjeto de aprendizagem (Canto, Lima e Tarouco, 2011), pois sua concepção tem como premissa "utilizar a multimodalidade na análise da multimodalidade". O acesso a tal metaobjeto é realizado através da internet, utilizando browsers compatíveis com a tecnologia Microsoft Silverligh (RIA - Rich Internet Application).

A especificação e desenvolvimento do aplicativo utilizaram os princípios de projeto propostos por Canto et. al.(2012); suas telas apresentam elementos verbais (textos escritos e narrações) e não verbais (imagens, animações, efeitos sonoros). A classificação dos objetos é feita a partir de uma sequência de perguntas com respostas binárias (sim/não). O diagnóstico necessita que sejam respondidas de uma a sete perguntas. A Figura 4 apresenta o caso mais rápido (diagnóstico após uma resposta), quando o objeto analisado não possui o modo verbal.

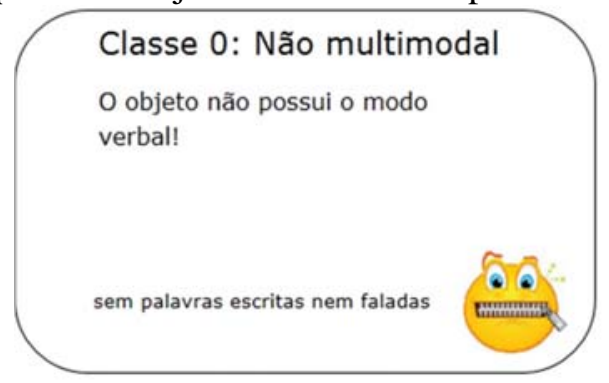

Figura 4. Não multimodal

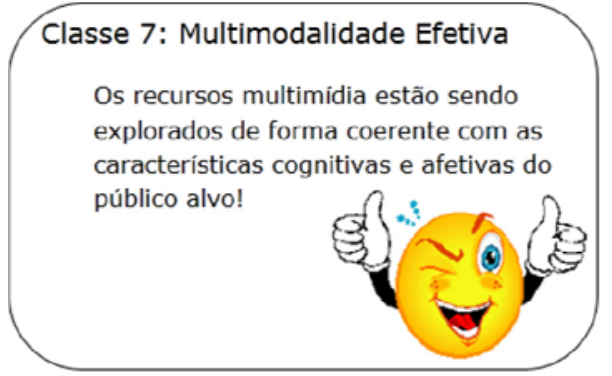

Figura 5. Multimodalidade Efetiva 
A classificação de Multimodalidade Efetiva (Figura 5) é a que requer o maior número de respostas (sete).

O fluxograma apresentado na Figura 3 mostra uma sequência de avaliação tal que, quando o OA puder ser enquadrado em mais de uma classe, ele ficará enquadrado na classe de menor efetividade. Por exemplo, caso um objeto possua redundância verbal (classe 5) e também seja dispersivo (classe 1), ele ficará enquadrado na classe 1.

\section{Resultados e Discussões}

Com o objetivo de realizar uma avaliação preliminar do método de análise proposto, os autores utilizaram o aplicativo desenvolvido para analisar cinco OAs quanto à multimodalidade. As seções a seguir apresentam as análises realizadas.

\section{1. $\mathrm{E}_{2} \mathrm{D}$ - Ensino de Derivadas a Distância}

$\mathrm{O} \mathrm{E}_{2} \mathrm{D}^{1}$ é uma ferramenta criada por professores das áreas de Matemática e Informática, alunos de um programa de Doutorado em Informática na Educação, com vistas a promover um maior aprendizado de Cálculo e, mais especificamente, de derivadas. Apresenta um conjunto de seções distintas, a saber: Apresentação, Prérequisitos e Revisão, Conceituação, Regras de Derivação e Atividades Práticas. Sua interface pode ser vista na Figura 6.

Com relação à classificação de multimodalidade, foco desta pesquisa, podese dizer que $\mathrm{o} \quad \mathrm{E}_{2} \mathrm{D}$ classifica-se, predominantemente, na Classe 2 (não multimídia), uma vez que elementos sonoros, sejam eles verbais ou não, são utilizados em poucos momentos, são exploradas predominantemente informações gráficas e textuais. Porém, nestes momentos em que tais elementos são utilizados, não é caracterizada uma redundância verbal, pois não é narrado o texto que se encontra na tela.

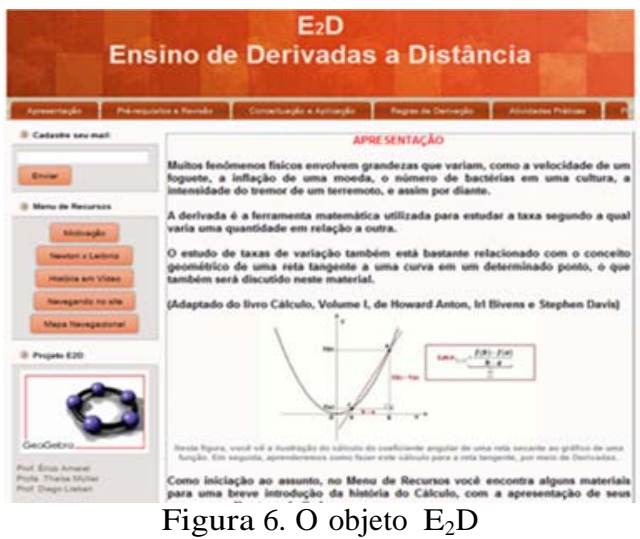
Sendo assim, nas telas em que existe um áudio, há uma oscilação entre as classes quatro (Texto Sonorizado Ilustrado) e sete (Multimídia Efetivo).

\subsection{Wikipédia - Sistema de Numeração Decimal}

Neste caso, foi realizada a análise de uma página da Wikipédia sobre o sistema de numeração decimal ${ }^{2}$, apresentada na Figura 7.

Disponível em: ${ }^{1}$ http://cesta2.cinted.ufrgs.br/xmlui/handle/123456789/675

2 http://pt.wikipedia.org/wiki/Sistema_de_numera\%C3\%A7\%C3\%A3o_decimal 


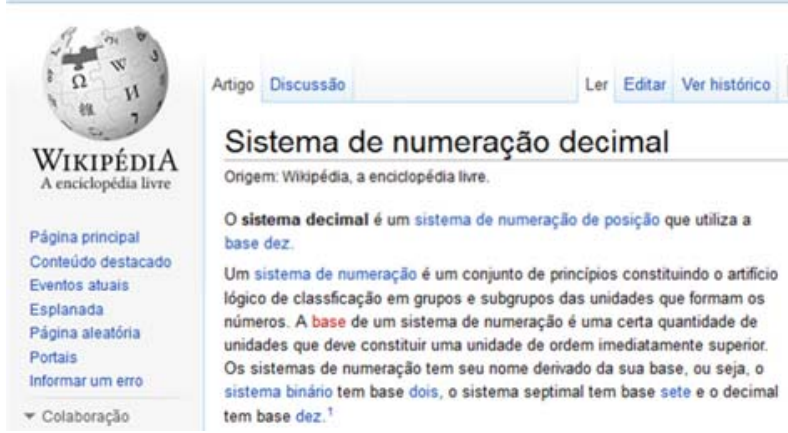

Figura 7. Wikipédia - Sistema de Numeração Decimal
Este material foi classificado como não multimodal, tendo em vista que toda a informação está apresentada como um texto escrito. Observa-se, no entanto, que existem algumas sutilezas que podem afetar o resultado, dependendo da percepção do avaliador. Nesta página existe uma imagem (logotipo da Wikipédia) que, dependendo do avaliador, poderá considerar que: (a)

não pertence ao OA, pois se trata apenas da moldura. (b) pertence ao OA mas é neutro (não ajuda nem atrapalha o aprendizado); (c) pertence ao OA e é dispersivo por não possuir identidade com o objetivo educacional; (d) pertence ao OA e é um elemento de design que torna o trabalho mais agradável sem dispersar a atenção.

\subsection{Vídeo Instrucional}

O objeto analisado nesta seção é uma vídeo-aula sobre limites ${ }^{3}$.

Neste objeto, observa-se claramente a existência de elementos verbais no canal de áudio e elementos verbais e não verbais apresentados na tela. Observa-se também que não ocorre redundância verbal (narração do texto apresentado na tela). $\mathrm{Na}$ avaliação dos autores, este objeto foi enquadrado na classe 7 - multimodal efetivo. Assumiu-se que o grande número de acessos no youtube é uma

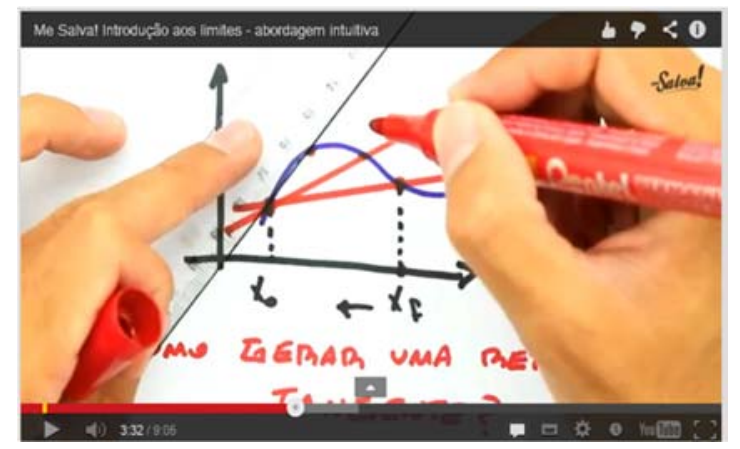

Figura 8. Me Salva - Vídeo sobre Limites evidência de sua capacidade de atrair a atenção dos estudantes, isto é, a dimensão afetiva foi contemplada adequadamente. No entanto, também este objeto possui particularidades que poderiam levar a classificações distintas: (a) a utilização do youtube como canal de divulgação implica a apresentação mensagens comerciais, que são elementos dispersivos; (b) seria o número de acessos um indicador adequado para avaliar a dimensão afetiva?

\subsection{Objeto Interativo}

O objeto NumBin ${ }^{4}$, cuja análise é apresentada nesta seção, destina-se ao aprendizado de sistemas de numeração e números binários. Observa-se que o objeto possui diversos segmentos com características distintas, razão pela qual esta análise não pode ser realizada para o objeto como um todo. Foram analisados dois segmentos: (a) o primeiro segmento, correspondente ao menu "Base Dez", que apresenta uma animação sobre a criação dos números na base dez, classificado como multimodal efetivo; (b) a forma como as questões (Figura 9) são apresentadas, classificado como Redundância Verbal.

\footnotetext{
${ }^{3}$ http://www.mesalva.com/\#/video/162

${ }^{4}$ Demonstração disponível em chasqueweb.ufrgs.br/ alberto.canto/NumBin/
} 
O aspecto a ser destacado nesta análise é a confirmação do fato de que nem sempre é possível analisar um OA como um todo, o que leva a uma análise "por segmento". Este objeto em particular foi objeto de pesquisas anteriores nas quais alguns alunos apresentaram manifestações contraditórias à animação apresentada na primeira parte: enquanto alguns estudantes manifestaram-se entusiasmados, outros julgaram que a mesma poderia ser descartada do OA. Uma investigação mais apurada sobre estas

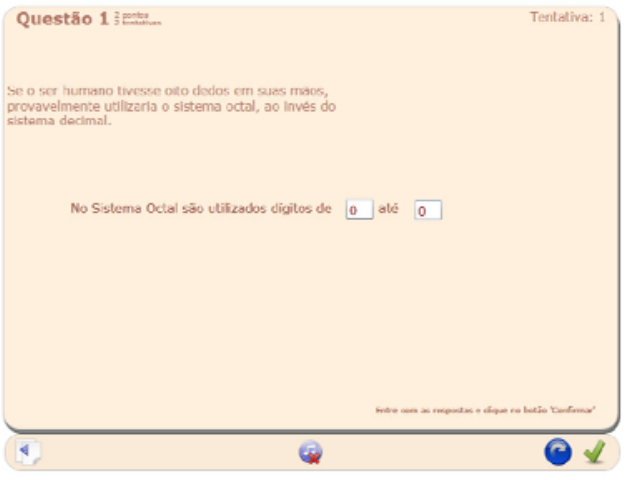

Figura 9. Questão 1 - NumBin divergências encontra-se em andamento.

\subsection{Cisco Packet Tracer}

Este é um Objeto de Aprendizagem desenvolvido para o ensino na área de redes de computadores $^{5}$. Esta ferramenta suporta uma vasta gama de simulações físicas e lógicas, além de fornecer recursos de visualização para ajudar a entender o comportamento dos elementos em uma rede. A Figura 10 apresenta a tela de acesso à aplicação.

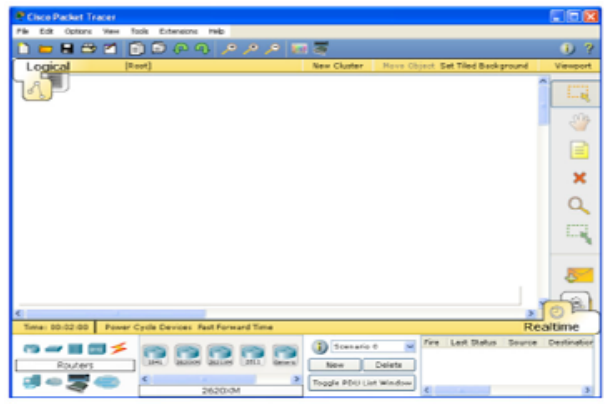

Figura 10. Cisco Packet Tracer Ao observar o Cisco Packet Tracer (CPT) e responder à aplicação MCS, inicialmente foi possível classificar este OA como Classe 2, não multimídia. A inserção do CPT nesta classe dá-se pelo fato da ausência de áudio no software, o que o caracteriza com baixo uso de recursos multimídia. Apesar disso, o CPT é uma ferramenta de aprendizagem que suporta uma vasta gama de simulações físicas e lógicas de ambientes de redes, provocando uma experiência real ao aluno e permitindo

um alto grau de interatividade.

\section{Conclusões}

Este artigo propõe um sistema de classificação quanto à multimodalidade, o qual admite que um mesmo objeto seja enquadrado em mais de uma classe, razão pela qual foi definido também um procedimento de classificação que garanta que, quando houver ambiguidades, o objeto seja enquadrado na classe de menor efetividade. Este procedimento foi automatizado no aplicativo chamado MCS - Multimodality Classification System, que por sua vez foi utilizado para analisar alguns OAs. As análises realizadas apresentaram resultados dentro das expectativas dos autores. Observa-se, no entanto, que alguns dos objetos são segmentados, muitas vezes adotando estratégias de apresentação diferentes em cada segmento. Nestes casos é inviável a classificação do OA como um todo, recomendando-se que cada segmento seja classificado separadamente.

Alguns aspectos considerados no sistema de avaliação proposto são atributos intrínsecos do OA e podem ser avaliados de forma objetiva ou subjetiva. Mais do que

\footnotetext{
${ }^{5}$ Disponível em http://www.cisco.com/web/learning/netacad/course_catalog/PacketTracer.html.
} 
uma ferramenta de diagnóstico, o aplicativo MCS pode ser considerado um objeto de aprendizagem a ser utilizado com objetivos educacionais relacionados ao ensino e aprendizagem da multimodalidade.

O uso continuado do MCS mostra que as animações e áudios se tornam cansativos e desnecessários à medida que os conceitos são dominados, confirmando as observações de van Merrënboer e Sweller (2010), de que a multimodalidade pode ser contraproducente quando já existe domínio do assunto. A partir desta observação, especificou-se uma nova versão do aplicativo de diagnóstico, na qual o usuário poderá configurar o modo de operação aprendiz ou revisão.

\section{Referências}

AUSUBEL, D. P.; NOVAK, J. D.; HANESIAN, H. Psicologia educacional, 2nd ed. Rio de Janeiro, Brasil: Interamericana, 1978.

BADDELEY, A. D. Working memory. Current Biology, vol. 23 (4), pp. 136-140, 2010.

BATTISTELlA, P. E. et al., Classificação de Objetos de Aprendizagem e análise de Ferramentas de Autoria, In: XX Simpósio Brasileiro de Informática na Educação, Florianópolis-SC, 2009.

BLOOM, B. S. Taxonomy of educational objectives: the classification of educational goals. New York: Longmans, 1956.

CANTO, A. B.; LIMA, J. V.; BERCHT, M.; TAROUCO, L. M. R. Recomendações de projeto de objetos de aprendizagem: em busca da autonomia na aprendizagem. In: TISE - Congreso Internacional de Informática Educativa, Santiago - Chile, 2012, pp. 315-323.

CANTO, A. B.; LIMA, J. V.; TAROUCO, L.M.R. Metaobjetos de Aprendizagem, RENOTE - Revista Novas Tecnologias na Educação, vol. 9, pp. 1-10, 2011.

CANTO, A. B.; MÜLleR, T. J.; AMARAL, E. M. H.; LIMA, J. V.; TAROUCO, L. M. R. Classificação de Objetos de Aprendizagem Segundo o Grau de Multimodalidade, RENOTE - Revista Novas Tecnologias na Educação, vol. 11, pp. 1-10, 2013.

FELDER, R. M.; BRENT, R. Understanding student differences. Journal of Engineering Education, vol. 1, pp. 57-72, 2005.

GRIMLEY, M.; RIDING. R. Individual differences and web-based learning. In: Cognitive and emotional processes in web-based education: integrating human factors and personalization. Hershey: IGI Global, 2009, pp. 209-228.

LOW, R.; SWELLER, J. The modality principle in multimedia learning. In: Cambridge handbook of multimedia learning. New York: Cambridge University Press, 2005, pp. 147-158.

MACÊDO, L. N.; MACÊDO, A. A. M.; FILHO, J. A. C. Avaliação de um Objeto de Aprendizagem com Base nas Teorias Cognitivas. In: XIII Workshop sobre Informática na Escola, XXVII Congresso SBC, Rio de Janeiro-RJ, 2007.

MAYER, R. E. Introduction to multimedia learning. In: The Cambridge Handbook of Multimedia Learning. New York: Cambridge University Press, 2005.

MAYER, R. E.; HEISER, J.; LONN, S. Cognitive Constraints on Multimedia Learning: When Presenting More Material Results in Less Understanding. Journal of Educational Psychology, vol. 93(1), pp. 187198, 2001.

PAAS, F.; RENKL, A.; SWELLER, J. Cognitive Load Theory and Instructional Design: Recent Developments. Educational Psychologist, vol. 38, 2003.

PAIVIO, A. Mental representations: A dual coding approach. Oxford, England: Oxford University Press, 1986.

RIDING, R.; CHEEMA, I. Cognitive style - an overview and integration. Educational Psychology, vol. 11, pp. 193-215, 1991.

SUNG, E.; MAYER, R. E. When graphics improve liking but not learning from online lessons. Computers in Human Behavior, vol. 28, pp. 1619-1625, May 2012.

SWELLER, J. Cognitive load during problem solving: Effects on learning. Cognitive Science, vol. 12, pp. 257-285, 1988.

SWELLER, J. et. al. Cognitive architecture and instructional design. Educational Psychology Review, vol. 10, pp. 251-296, 1998.

VAN MERRIËNBOER, J. J. G.; SWELLER, J. Cognitive load theory in health professional education: design principles and strategies. Medical Education, vol. 44, pp. 85-93, 2010. 